\title{
Performance evaluation of adiabatic quantum computation via quantum speed limits and possible applications to many-body systems
}

\author{
Keisuke Suzuki \\ Department of Physics, Tokyo Institute of Technology, Tokyo 152-8551, Japan \\ Kazutaka Takahashi () \\ Institute of Innovative Research, Tokyo Institute of Technology, Kanagawa 226-8503, Japan
}

(Received 7 January 2020; revised 12 April 2020; accepted 24 June 2020; published 15 July 2020)

\begin{abstract}
The quantum speed limit specifies a universal bound of the fidelity between the initial state and the timeevolved state. We apply this method to find a bound of the fidelity between the adiabatic state and the timeevolved state. The bound is characterized by the counterdiabatic Hamiltonian and can be used to evaluate the worst case performance of the adiabatic quantum computation. The result is improved by imposing additional conditions and we examine several models to find a tight bound. We also derive a different type of quantum speed limit that is meaningful even when we take the thermodynamic limit. By using solvable spin models, we study how the performance and the bound are affected by phase transitions.
\end{abstract}

DOI: 10.1103/PhysRevResearch.2.032016

Introduction. Knowing the fundamental speed limit for a dynamical process is an important problem in physics and is relevant to a broad range of research fields. Recent advances in quantum control technologies allow us to discuss the fundamental limit even from a practical point of view. In closed quantum systems, we can derive several limits known as quantum speed limits (QSLs) [1-5].

Among many possible applications [6-14], we focus our attention on adiabatic quantum computation (AQC). It is a method solving combinatorial optimization problems and has attracted intensive attention recently, as quantum annealing [15-19], due to its use for a device manufactured by D-Wave Systems, Inc. [20,21]. The solution of the problem is set to the ground state of the Ising Hamiltonian. The Hamiltonian is slowly changed from a trivial form, represented by the transverse-field term, to the Ising Hamiltonian. If the rate of the Hamiltonian change is very small, the time-evolved state is close to the instantaneous ground state of the Hamiltonian.

The principle of AQC is based on the adiabatic theorem. The infinitely slow time evolution is not realistic and we find nonadiabatic transitions in experiments. Therefore, estimating and suppressing errors are important not only for its general use but also for understanding the dynamical properties of the quantum systems. In the method of quantum adiabatic brachistochrone, a cost function is defined based on the notion of adiabaticity [22-24]. It is minimized with respect to the protocol to obtain an optimized algorithm. The method practically gives a good performance but the result is strongly

Published by the American Physical Society under the terms of the Creative Commons Attribution 4.0 International license. Further distribution of this work must maintain attribution to the author(s) and the published article's title, journal citation, and DOI. dependent on the choice of the cost function and does not assess the quantitative performance. Some rigorous treatment of the adiabatic theorem allows us to derive bounds of the performance [25-28], but mathematically involved approaches are required for the derivation and the result is of limited use because of the complicated expressions of the bound.

To overcome these problems, we employ the theory of QSL to find a rigorous bound. The QSL has a geometrical meaning and we can find a universal bound by using this approach. Since the standard QSL only requires several simple inequalities, the derivation is simple and the result can be written in an intuitively understandable form. The QSL can give us a tight bound, which is also useful for practical applications such as the AQC. We can further use the bound as a cost function to optimize the AQC.

We treat closed quantum systems throughout this Rapid Communication. For a given Hamiltonian $H(t)$ and a initial state $|\psi(0)\rangle$, unitary Schrödinger dynamics yields a timeevolved state $|\psi(t)\rangle$. It satisfies the Mandelstam-Tamm (MT) relation [1-4]

$$
\arccos |\langle\psi(0) \mid \psi(T)\rangle| \leqslant \int_{0}^{T} d t \sigma[H(t),|\psi(t)\rangle],
$$

where $\sigma(H,|\psi\rangle)=\sqrt{\left\langle\psi\left|H^{2}\right| \psi\right\rangle-\langle\psi|H| \psi\rangle^{2}}$. The left-hand side of Eq. (1) represents the Fubini-Study angle and is used as a natural measure of the state separation. It takes a positive value between 0 and $\pi / 2$. Equation (1) shows that the angle has a bound characterized by the energy variance. Since the angle is interpreted as a distance measure, $\sigma$ plays a role of velocity. This relation results from a general property of vectors in Hilbert space. Applying a Hermitian operator $H$ to a state vector $|\psi\rangle$ gives

$$
H|\psi\rangle=|\psi\rangle\langle\psi|H| \psi\rangle+\left|\psi_{\perp}\right\rangle \sigma(H,|\psi\rangle),
$$


where $\left|\psi_{\perp}\right\rangle$ is a normalized state orthogonal to $|\psi\rangle$. The second term represents how the state deviates from the original one and is the origin of the bound in the MT relation.

Quantum speed limit for adiabatic quantum computation. In the AQC, we are interested in obtaining the instantaneous ground state of the time-dependent Hamiltonian $H(t)$ by Schrödinger dynamics. A slow driving approximately gives the adiabatic state $\left|\psi_{\text {ad }}(t)\right\rangle$ whose formal definition is given in the following. The performance of the computation is evaluated by the fidelity between the ideal adiabatic state and the time-evolved state $|\psi(t)\rangle$,

$$
\theta_{\mathrm{ad}}(t)=\arccos \left|\left\langle\psi_{\mathrm{ad}}(t) \mid \psi(t)\right\rangle\right| .
$$

When we write the adiabatic state as a unitary time evolution as $\left|\psi_{\mathrm{ad}}(t)\right\rangle=U_{\mathrm{ad}}(t)|\psi(0)\rangle$, the overlap is written as $\left\langle\psi_{\mathrm{ad}}(t) \mid \psi(t)\right\rangle=\langle\psi(0) \mid \tilde{\psi}(t)\rangle$, where $|\tilde{\psi}(t)\rangle=U_{\mathrm{ad}}^{\dagger}(t)|\psi(t)\rangle$. The formal expression of the unitary operator $U_{\mathrm{ad}}(t)$ is given by

$$
U_{\mathrm{ad}}(t)=\sum_{n} e^{-i \int_{0}^{t} d t^{\prime} \epsilon_{n}\left(t^{\prime}\right)-\int_{0}^{t} d t^{\prime}\left\langle n\left(t^{\prime}\right) \mid \dot{n}\left(t^{\prime}\right)\right\rangle}|n(t)\rangle\langle n(0)|,
$$

where $\{|n(t)\rangle\}$ represents a set of instantaneous eigenstates of $H(t)$ with the corresponding eigenvalues $\left\{\epsilon_{n}(t)\right\}$ and the dot denotes the time derivative. The time derivative of $U_{\mathrm{ad}}(t)$ gives

$$
i \frac{d U_{\mathrm{ad}}(t)}{d t}=\left[H(t)+H_{\mathrm{CD}}(t)\right] U_{\mathrm{ad}}(t),
$$

where

$$
H_{\mathrm{CD}}(t)=i \sum_{n}(1-|n(t)\rangle\langle n(t)|)|\dot{n}(t)\rangle\langle n(t)| .
$$

$H_{\mathrm{CD}}(t)$ is known as the counterdiabatic term in the method of shortcuts to adiabaticity (STA) [29-34]. By adding this term to the original Hamiltonian, we can realize the adiabatic state of the original Hamiltonian exactly by the time evolution. Using this result, we find that the generator of the state $|\tilde{\psi}(t)\rangle$ is given by $\tilde{H}(t)=-U_{\mathrm{ad}}^{\dagger}(t) H_{\mathrm{CD}}(t) U_{\mathrm{ad}}(t)$. Then, we can immediately apply the MT relation to obtain the bound

$$
\begin{aligned}
\theta_{\mathrm{ad}}(T) \leqslant & \int_{0}^{T} d t\left|\dot{\theta}_{\mathrm{ad}}(t)\right| \\
\leqslant & \min \left(\int_{0}^{T} d t \sigma\left[H_{\mathrm{CD}}(t),|\psi(t)\rangle\right],\right. \\
& \left.\int_{0}^{T} d t \sigma\left[H_{\mathrm{CD}}(t),\left|\psi_{\mathrm{ad}}(t)\right\rangle\right]\right) .
\end{aligned}
$$

The bound is characterized by two types of variance. Since the counterdiabatic term is represented by using the time derivative of parameters in the original Hamiltonian, it is natural for this term to characterize the bound. We can take the minimum of the variances to obtain a tight bound. When the Hamiltonian $H(t)$ is prepared and we do not know the ideal adiabatic state $\left|\psi_{\text {ad }}(t)\right\rangle$, the bound by the realistic state $|\psi(t)\rangle$ can be useful. On the other hand, when we prepare $\left|\psi_{\text {ad }}(t)\right\rangle$ as a reference state, the bound by $\left|\psi_{\text {ad }}(t)\right\rangle$ would be appropriate. The choice of the Hamiltonian $H(t)$ that corresponds to the specified state $\left|\psi_{\text {ad }}(t)\right\rangle$ is not unique and we can obtain a universal bound which is common to all possible choices. The variance with respect to $\left|\psi_{\text {ad }}(t)\right\rangle$ has a geometrical meaning and appears when we discuss an energetic cost and a tradeoff relation for the implementation of the counterdiabatic term [35-38]. In the following examples, we study bounds by $|\psi(t)\rangle$ since they give the same or slightly better results compared to those by $\left|\psi_{\text {ad }}(t)\right\rangle$.

In the theory of QSL, we are basically interested in maximizing the left-hand side of Eq. (1). The MT relation shows that the maximum possible speed is given by the energy variance. Here, we want to minimize $\theta_{\mathrm{ad}}(T)$, which means that the speed limit, the rightmost side in Eq. (7), gives a worst case evaluation of the performance. Minimizing the variance can be an optimization method for the AQC. In fact, the method of quantum adiabatic brachistochrone introduces a similar quantity for an optimization [22-24].

In the AQC, we expect that $\theta_{\mathrm{ad}}(t)$ is small and $\dot{\theta}_{\mathrm{ad}}(t)$ oscillates around zero. The original MT relation in Eq. (1) gives a tight bound only when $\arccos |\langle\psi(0) \mid \psi(t)\rangle|$ is a monotonic function. The same is true for $\theta_{\mathrm{ad}}(t)$ in Eq. (7) and the equality is unlikely to hold in the present situation. If we strictly impose the adiabaticity of the computation, the intermediate state $|\psi(t)\rangle$ at arbitrary $t$ is expected to be close to the adiabatic state $\left|\psi_{\text {ad }}(t)\right\rangle$. Basically, we are interested in the final state and it is not necessary for the intermediate state to satisfy the adiabaticity. However, we expect that an adiabatic-state following leads to a robust computation. Then, $\int_{0}^{T} d t\left|\dot{\theta}_{\text {ad }}(t)\right|$, the middle term in Eq. (7), becomes a proper measure of adiabaticity and the rightmost side in Eq. (7) can be a tight bound for this improved measure as we see in the following.

It is often a difficult task to calculate the explicit form of the bound. The present result shows that the bound is directly connected to the counterdiabatic term. We know various ways to construct the counterdiabatic term exactly [39-42] and approximately $[43,44]$, which would be useful to estimate the bound.

Some improvements. The bound can be improved by imposing additional conditions. One of the simplest conditions is to set that the initial state $|\psi(0)\rangle$ is one of the eigenstates of the initial Hamiltonian $H(0),|n(0)\rangle$. This is a natural condition usually employed in the AQC. In this case, the adiabatic state is written by the single eigenstate $|n(t)\rangle$. The dynamical phase factor $e^{-i \int_{0}^{t} d t^{\prime} \epsilon_{n}\left(t^{\prime}\right)}$ gives an overall contribution and is dropped out when we take the absolute value of the overlap. The time evolution is effectively achieved only by the counterdiabatic term, that means $U_{\mathrm{ad}}(t)$ is equivalent to $e^{-i \int_{0}^{t} d t^{\prime} \epsilon_{n}\left(t^{\prime}\right)} U_{\mathrm{CD}}(t)$ where $U_{\mathrm{CD}}(t)$ is the time-evolution operator for $H_{\mathrm{CD}}(t)$ and is obtained by setting $\epsilon_{n}(t)=0$ for $U_{\mathrm{ad}}(t)$ in Eq. (4). We find that the bound is obtained by replacing $\sigma\left[H_{\mathrm{CD}}(t),|\psi(t)\rangle\right]$ in Eq. (7) by $\sigma\left[H(t)-H_{\mathrm{CD}}(t),|\psi(t)\rangle\right]$. Although this bound is expected to be an improvement over that in Eq. (7), it is not evident whether $\sigma\left[H(t)-H_{\mathrm{CD}}(t),|\psi(t)\rangle\right]$ is smaller than $\sigma\left[H_{\mathrm{CD}}(t),|\psi(t)\rangle\right]$. To obtain some intuition, we find a further different expression in the following.

The idea also comes from the method of STA. In the counterdiabatic driving, we introduce the additional counterdiabatic term $H_{\mathrm{CD}}(t)$ to the original Hamiltonian $H(t)$ to keep the adiabatic state with respect to $H(t)$. The idea of STA is not restricted to this procedure and we can consider several variants of implementations. In fact, in the "inverse engineering," 
we use the dynamical invariant operator to obtain an ideal time evolution $[32,45]$. From a viewpoint of the counterdiabatic driving, the use of the dynamical invariant corresponds to decomposing the Hamiltonian into two parts: $H(t)=H_{0}(t)+$ $H_{1}(t)$. The first term $H_{0}(t)$ commutes with the dynamical invariant. The solution of the Schrödinger equation with $H(t)$ is given by the adiabatic state of $H_{0}(t)$, which means that $H_{1}(t)$ is interpreted as the counterdiabatic term. $H_{1}(t)$ is different from $H_{\mathrm{CD}}(t)$ as we discuss below. When we start the time evolution from an eigenstate of the initial Hamiltonian, we determine the decomposition as follows. We prepare the basis $\{U(t)|n(0)\rangle\}$, where $U(t)$ is the time-evolution operator for $H(t)$. This basis represents a set of eigenstates for the dynamical invariant. Then, $H_{0}(t)$ represents the diagonal part and $H_{1}(t)$ the off-diagonal part [46]. Setting the initial state as $|\psi(0)\rangle=|n(0)\rangle$ and following the same logic as before, we see that $|\psi(t)\rangle$ is obtained, up to the phase, by applying the unitary operator $U_{1}(t)$ for $H_{1}(t)$ to the initial state. Then, we obtain the bound with $\sigma\left[H_{1}(t)-H_{\mathrm{CD}}(t),|\psi(t)\rangle\right]$ in place of $\sigma\left[\left(H(t)-H_{\mathrm{CD}}(t),|\psi(t)\rangle\right]\right.$.

Adiabatic expansion. The representation of the bound using the difference between two counterdiabatic terms is instructive. Although the explicit operator form of $H_{1}(t)$ is generally hard to obtain, we can find an intuitive meaning by using the adiabatic expansion. The dynamical invariant operator $F(t)$ satisfies

$$
i \frac{d F(t)}{d t}=[H(t), F(t)],
$$

and is solved formally by using the expansion in terms of the time derivative operator. Since the dynamical invariant commutes with $H_{0}(t), H_{1}(t)$ is obtained by solving the commutation relation $\left[H(t)-H_{1}(t), F(t)\right]=0$. Solving these equations order by order, we find

$$
\begin{aligned}
\left\langle m(t)\left|H_{1}(t)\right| n(t)\right\rangle= & \left\langle m(t)\left|H_{\mathrm{CD}}(t)\right| n(t)\right\rangle \\
& +i \frac{d}{d t} \frac{\left\langle m(t)\left|H_{\mathrm{CD}}(t)\right| n(t)\right\rangle}{\epsilon_{m}(t)-\epsilon_{n}(t)}+\cdots,
\end{aligned}
$$

for $m \neq n$. The diagonal part $\left\langle n(t)\left|H_{1}(t)\right| n(t)\right\rangle$ is not required when we calculate the variance. The result shows that $H_{1}(t)$ is equivalent to $H_{\mathrm{CD}}(t)$ at first order of the expansion. We use the variance of $H_{1}(t)-H_{\mathrm{CD}}(t)$ for the bound, which means that the bound can be approximately characterized by the second term of Eq. (9) in the adiabatic regime. We obtain

$$
\begin{aligned}
& \sigma^{2}\left[H_{1}(t)-H_{\mathrm{CD}}(t),|\psi(t)\rangle\right] \\
& \quad \sim \sum_{m(\neq n)}\left|\frac{d}{d t}\left(\frac{\left\langle m(t)\left|H_{\mathrm{CD}}(t)\right| n(t)\right\rangle}{\epsilon_{m}(t)-\epsilon_{n}(t)}\right)\right|^{2} .
\end{aligned}
$$

Since $H_{\mathrm{CD}}(t)$ incorporates the time derivative of the parameters, the second time derivative of the parameters is relevant, rather than the first derivative, for characterizing the bound of the fidelity. The acceleration can be relevant for some control problems. In the counterdiabatic driving, the acceleration potential is obtained by using a unitary transformation [47]. The relevance of the higher-order derivatives for the adiabatic approximation can also be seen in rigorous treatments of the adiabatic theorem [19,25-28].

Two-level systems. We study a simple two-level system to see how tight the obtained bounds are. The Hamilto-
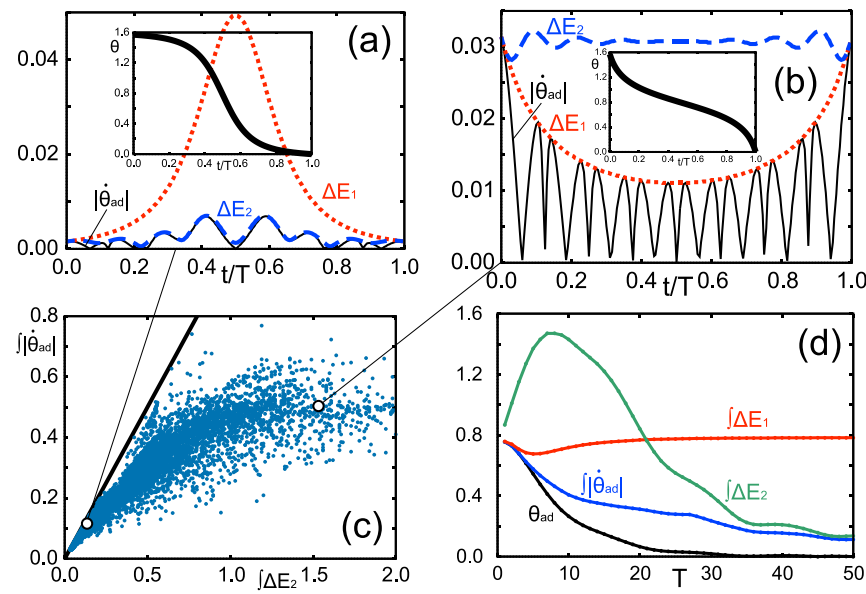

FIG. 1. QSL for a two-level system. (a), (b) $\left|\dot{\theta}_{\text {ad }}(t)\right|$ (black solid), $\Delta E_{1}(t)$ (red dotted), and $\Delta E_{2}(t)$ (blue dashed) for a protocol $\theta(t)$ given in the inset of each panel. We set the annealing time $T=50$ in units of $h$. (c) Distribution of $\left(\int_{0}^{T} d t \Delta E_{2}(t), \int_{0}^{T} d t\left|\dot{\theta}_{\mathrm{ad}}(t)\right|\right)$ for randomly generated protocols $\theta(t)$. The protocols are generated as decreasing functions from $\pi / 2$ to 0 . The solid line represents the bound. (d) Annealing time dependence for the protocol in the top left-hand panel. $\dot{\theta}_{\mathrm{ad}}(t)$ (black), $\left|\dot{\theta}_{\mathrm{ad}}(t)\right|$ (blue), $\Delta E_{1}(t)$ (red), and $\Delta E_{2}(t)$ (green) are integrated from 0 to $T$.

nian is given by $H(t)=\frac{h}{2}\left[\sigma^{z} \cos \theta(t)+\sigma^{x} \sin \theta(t)\right]$, where $\sigma^{z}\left(\sigma^{x}\right)$ is the $z(x)$ component of the Pauli matrices. $h$ is fixed to a constant value and $\theta(t)$ moves from $\theta(0)=\pi / 2$ to $\theta(T)=0$. In this case, $H_{\mathrm{CD}}(t)$ is given by $H_{\mathrm{CD}}(t)=\frac{\dot{\theta}(t)}{2} \sigma^{y}$. We also find $\sigma\left[H(t)-H_{\mathrm{CD}}(t),|\psi(t)\rangle\right]=$ $\sigma\left[H_{1}(t)-H_{\mathrm{CD}}(t),|\psi(t)\rangle\right]$. Then, we compare $\Delta E_{1}(t)=$ $\sigma\left[H_{\mathrm{CD}}(t),|\psi(t)\rangle\right]$ and $\Delta E_{2}(t)=\sigma\left[H(t)-H_{\mathrm{CD}}(t),|\psi(t)\rangle\right]$ as possible bounds.

The numerical study is summarized in Fig. 1. $\left|\dot{\theta}_{\mathrm{ad}}(t)\right|$ becomes small when the parameter changes around the initial and final times are slow, as we see in the top left-hand panel of Fig. 1. In this case, $\Delta E_{2}(t)$ gives a good tight bound. The importance of the slow changes at the boundaries has been discussed in several works [26,48,49]. Our observation is consistent with their results. It should be remarked that we can find a good bound irrespective of the performance. Although the oscillations of $\left|\dot{\theta}_{\mathrm{ad}}(t)\right|$ in the top right-hand panel are difficult to be captured by the bound, the bound by $\Delta E_{1}(t)$ can describe the outline of the oscillation as an envelope.

The annealing-time dependence of the result is shown in the bottom right-hand panel of Fig. 1. $\Delta E_{2}(t)$ is strongly dependent on the annealing time $T$ while $\Delta E_{1}(t)$ is not sensitive to $T . \Delta E_{2}(t)$ becomes small at large $T$, which is understood from the adiabatic expansion as discussed in Eq. (9).

Quantum speed limits for many-body systems. In the AQC, our interest is mainly on systems with many degrees of freedom. Although the obtained bounds are applicable to any closed quantum systems, they are not useful in typical manybody systems. For a system with the particle number $N$, the Hamiltonian is an extensive quantity and the state is basically given by a product of $N$ components. This means that the 
fidelity is expected to have a form

$$
\langle\psi(0) \mid \psi(t)\rangle \sim e^{-N g(t)},
$$

where $g(t)$ is a non-negative function independent of $N$. This becomes a very small quantity for a large $N$. In other words, the size of the Hilbert space is too huge for two vectors to have a certain amount of overlap. The vanishing of the overlap can be found even when we consider a small perturbation. It is called the orthogonality catastrophe [50] and has recently been discussed from a viewpoint of the QSL [51]. The behavior of the fidelity for many-body systems can be studied by using the rate function $g(t)$. In fact, a dynamical singularity appears on this quantity for systems with quantum quench [52]. When the rate function becomes a well-defined quantity, the overlap immediately goes to zero at $N \rightarrow \infty$. Since $\sigma[H(t),|\psi(t)\rangle]$ is typically proportional to $\sqrt{N}$, the MT relation becomes a trivial one.

To find a meaningful relation, we reexamine the derivation of the MT relation. Using Eq. (2), we find

$$
\begin{aligned}
|\dot{g}(t)| & \leqslant \frac{\sigma[H(t),|\psi(t)\rangle]}{N}\left|\frac{\left\langle\psi(0) \mid \psi_{\perp}(t)\right\rangle}{\langle\psi(0) \mid \psi(t)\rangle}\right| \\
& =\frac{\sigma[H(t),|\psi(t)\rangle]}{\sqrt{N}} \frac{c_{\perp}(t)}{c(t)} .
\end{aligned}
$$

The equality is obtained when the ratio $\left\langle\psi(0) \mid \psi_{\perp}(t)\right\rangle /$ $\langle\psi(0) \mid \psi(t)\rangle$ becomes pure imaginary. When the fidelity is scaled as $|\langle\psi(0) \mid \psi(t)\rangle|=c(t) e^{-N g(t)}$, where $c(t)$ and $g(t)$ are non-negative and independent of $N$, we see below $\left|\left\langle\psi(0) \mid \psi_{\perp}(t)\right\rangle\right|=\sqrt{N} c_{\perp}(t) e^{-N g(t)}$, with $c_{\perp}(t)$ which is also non-negative and independent of $N$. As a result, we obtain the last expression in Eq. (12). The right-hand side remains finite even if we take the thermodynamic limit $N \rightarrow \infty$. Then, we can use this relation as another type of QSL for many-body systems. We note that this inequality makes sense even for small systems. Since the present relation does not require an additional inequality $|\langle\psi(0) \mid \psi(t)\rangle|^{2}+\left|\left\langle\psi(0) \mid \psi_{\perp}(t)\right\rangle\right|^{2} \leqslant$ 1 which is used to derive the MT relation, we expect that Eq. (12) gives a tighter bound.

It is not convenient to represent the bound by using the unknown state $\left|\psi_{\perp}(t)\right\rangle$. The bound can be represented by the counterdiabatic term. Setting the condition that the initial state is in one of the eigenstate of the initial Hamiltonian, we obtain $H_{1}(t)|\psi(t)\rangle=\left|\psi_{\perp}(t)\right\rangle \sigma[H(t),|\psi(t)\rangle]$ and

$$
|\dot{g}(t)| \leqslant \frac{1}{N}\left|\frac{\left\langle\psi(0)\left|H_{1}(t)\right| \psi(t)\right\rangle}{\langle\psi(0) \mid \psi(t)\rangle}\right| .
$$

Since the counterdiabatic term is expected to be an extensive operator, we see that the right-hand side remains finite even if we take the limit $N \rightarrow \infty$. It is interesting to see that the quantity appearing on the right-hand side represents the weak value [53].

In a similar way, for the fidelity with the adiabatic state, we define $g_{\text {ad }}(t)$ as $\left|\left\langle\psi_{\text {ad }}(t) \mid \psi(t)\right\rangle\right| \sim e^{-N g_{\text {ad }}(t)}$ to derive the bound

$$
\left|\dot{g}_{\text {ad }}(t)\right| \leqslant \frac{1}{N}\left|\frac{\left\langle\psi_{\mathrm{ad}}(t)\left|H_{\mathrm{CD}}(t)\right| \psi(t)\right\rangle}{\left\langle\psi_{\mathrm{ad}}(t) \mid \psi(t)\right\rangle}\right| .
$$

With an additional condition that the initial state is in one of the eigenstate of the initial Hamiltonian, we can replace
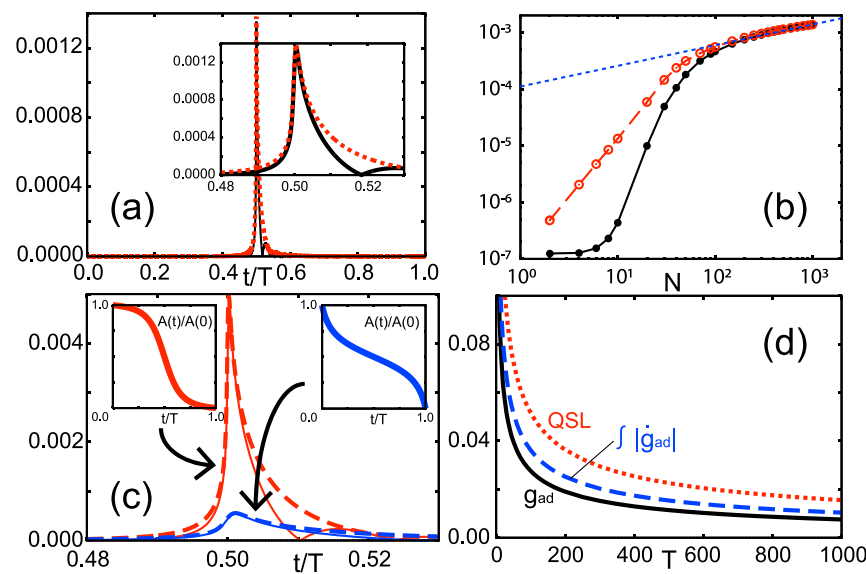

FIG. 2. QSL for a spin chain model. (a) $\left|\dot{g}_{\text {ad }}(t)\right|$ (black solid line) and the right-hand side of Eq. (14) (red dotted) for a linear protocol $A(t)=A(0)(1-t / T), B(t)=A(0) t / T$. We set $N=1000$ and $T=$ 1000 in units of $A(0)$. The inset represents a blowup around the peak. (b) Size dependence of the peak height obtained in (a). The black solid line with the symbol $\bullet$ denotes $\max _{t}\left|\dot{g}_{\text {ad }}(t)\right|$ and the red dashed line with $\circ$ the corresponding quantity in the QSL. They show the same power-law behavior denoted by the blue dotted line at large $N$. (c) Protocol dependence. We choose two types of $A(t)$ shown in the insets and $B(t)=A(0)-A(t)$. The solid lines denote $\left|\dot{g}_{\text {ad }}(t)\right|$ and the dashed lines the QSL. We set $N=1000$ and $T=1000$ in units of $A(0)$. (d) Annealing time dependence. We use the linear protocol used in (a) and $\operatorname{set} N=200$.

$H_{\mathrm{CD}}(t)$ in Eq. (14) by $H(t)-H_{\mathrm{CD}}(t)$ and $H_{1}(t)-H_{\mathrm{CD}}(t)$ as we have shown in the previous calculations.

Some examples. We study many-body spin models that exhibit phase transitions. First, we treat the transverse-field Ising-spin chain,

$$
H(t)=-\frac{A(t)}{2} \sum_{i=1}^{N} \sigma_{i}^{x}-\frac{B(t)}{2} \sum_{i=1}^{N} \sigma_{i}^{z} \sigma_{i+1}^{z} .
$$

We use the periodic boundary condition $\sigma_{N+1}^{z}=\sigma_{1}^{z}$. This Hamiltonian can be decomposed into a set of two-level systems by the Jordan-Wigner transformation [54,55]. The result is shown in Fig. 2. The QSL represented by Eq. (14) gives a tight bound even at the quantum phase-transition point $A=B$ obtained by the static treatment. We observe a peak at the point and the height is scaled by the size of the system as $N^{\alpha}$ with $\alpha \sim 0.303$ in the present choice of parameters. The same scaling is applied to both the fidelity and the QSL, which implies that the universal properties at the phase transition can be studied by using the QSL.

A different type of singularity can be found for a quantum quench system and is known as dynamical phase transitions [52]. We consider the spin operator $S$ with $S^{2}=\frac{N}{2}\left(\frac{N}{2}+1\right)$, and prepare the initial state $|\psi(0)\rangle$ as an eigenstate of $S^{x}$, $S^{x}|\psi(0)\rangle=\frac{N}{2}|\psi(0)\rangle$. Then, the state is time- evolved under the Hamiltonian

$$
H=-2\left(\frac{J}{N}\left(S^{z}\right)^{2}+h S^{z}\right) .
$$

It is known that the rate function $g(t)$ at $N \rightarrow \infty$ has singular points [56]. The decomposition of the Hamiltonian is 


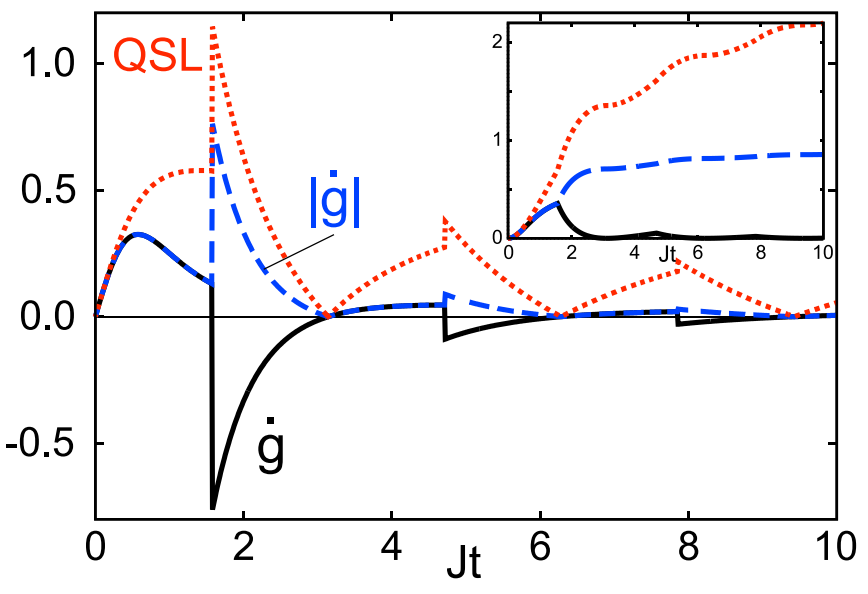

FIG. 3. QSL for a quantum quench system with dynamical phase transitions. We set $J=h$. The black solid line represents $\dot{g}(t)$, the blue dashed line $|\dot{g}(t)|$, and the red dotted line the QSL specified by the right-hand side in Eq. (13). Inset: Time-integrated quantities.

possible in this case [46] and we can calculate the bound as we show in Fig. 3. $\dot{g}(t)$ changes discontinuously at phase-transition points. We find that the QSL still holds even when we have the transitions. We also see that the bound can basically be a good estimate of the rate function but it becomes loose around the transition points. Since $H_{1}(t)$ is a part of the original Hamiltonian, the bound in Eq. (13) stays finite, which indicates that $g(t)$ does not diverge in any systems.

Conclusion. We have discussed the QSL applied to the AQC. The performance of the computation is characterized by the counterdiabatic term. The bound is simply represented by the variance of the counterdiabatic term and has a geometrical meaning. Although we mainly focused on the AQC, the result is general enough so that it can be used without any additional condition. As we mentioned in the Introduction, the present method makes up for the inconvenience of the previous methods. We also find another type of QSL that can be applied to many-body systems. Our result implies that the universal properties can be deduced from the corresponding counterdiabatic term.

Acknowledgments. We are grateful to Adolfo del Campo, Ken Funo, Takuya Hatomura, and Yutaka Shikano for useful discussions and comments.
[1] L. Mandelstam and I. Tamm, The uncertainty relation between energy and time in nonrelativistic quantum mechanics, J. Phys. (Moscow) 9, 249 (1945), in Selected Papers, edited by B. M. Bolotovskii, V. Y. Frenkel, and R. Peierls (Springer, Berlin, Heidelberg, 1991), pp. 115-123.

[2] G. N. Fleming, A unitary bound on the evaluation of nonstationary states, Nuovo Cimento A 16, 232 (1973).

[3] K. Bhattacharyya, Quantum decay and the Mandelstam-Tamm time-energy inequality, J. Phys. A 16, 2993 (1983).

[4] L. Vaidman, Minimum time for the evolution to an orthogonal quantum state, Am. J. Phys. 60, 182 (1992).

[5] N. Margolus and L. B. Levitin, The maximum speed of dynamical evolution, Physica D 120, 188 (1998).

[6] S. Lloyd, Ultimate physical limits to computation, Nature (London) 406, 1047 (2000).

[7] S. Lloyd, Computational Capacity of the Universe, Phys. Rev. Lett. 88, 237901 (2002).

[8] L. B. Levitin and T. Toffoli, Fundamental Limit on the Rate of Quantum Dynamics: The Unified Bound is Tight, Phys. Rev. Lett. 103, 160502 (2009).

[9] M. M. Taddei, B. M. Escher, L. Davidovich, and R. L. de Matos Filho, Quantum Speed Limit for Physical Processes, Phys. Rev. Lett. 110, 050402 (2013).

[10] A. del Campo, I. L. Egusquiza, M. B. Plenio, and S. F. Huelga, Quantum Speed Limits in Open System Dynamics, Phys. Rev. Lett. 110, 050403 (2013).

[11] S. Deffner and E. Lutz, Quantum Speed Limit for NonMarkovian Dynamics, Phys. Rev. Lett. 111, 010402 (2013).

[12] A. D. Cimmarusti, Z. Yan, B. D. Patterson, L. P. Corcos, L. A. Orozco, and S. Deffner, Environment-Assisted Speed-up of the Field Evolution in Cavity Quantum Electrodynamics, Phys. Rev. Lett. 114, 233602 (2015).
[13] S. Deffner and S. Campbell, Quantum speed limits: From Heisenberg's uncertainty principle to optimal quantum control, J. Phys. A: Math. Theor. 50, 453001 (2017).

[14] M. Bukov, D. Sels, and A. Polkovnikov, Geometric Speed Limit of Accessible Many-Body State Preparation, Phys. Rev. X 9, 011034 (2019).

[15] T. Kadowaki and H. Nishimori, Quantum annealing in the transverse Ising model, Phys. Rev. E 58, 5355 (1998).

[16] J. Brooke, D. Bitko, T. F. Rosenbaum, and G. Aeppli, Quantum annealing of a disordered magnet, Science 284, 779 (1999).

[17] E. Farhi, J. Goldstone, S. Gutmann, and M. Sipser, Quantum computation by adiabatic evolution, arXiv:quant-ph/0001106.

[18] E. Farhi, J. Goldstone, S. Gutmann, J. Lapan, A. Lundgren, and D. Preda, A quantum adiabatic evolution algorithm applied to random instances of an NP-complete problem, Science 292, 472 (2001).

[19] T. Albash and D. A. Lidar, Adiabatic quantum computation, Rev. Mod. Phys. 90, 015002 (2018).

[20] M. W. Johnson, M. H. S. Amin, S. Gildert, T. Lanting, F. Hamze, N. Dickson, R. Harris, A. J. Berkley, J. Johansson, P. Bunyk et al., Quantum annealing with manufactured spins, Nature (London) 473, 194 (2011).

[21] S. Boixo, T. F. Rønnow, S. V. Isakov, Z. Wang, D. Wecker, D. A. Lidar, J. M. Martinis, and M. Troyer, Evidence for quantum annealing with more than one hundred qubits, Nat. Phys. 10, 218 (2014).

[22] A. T. Rezakhani, W.-J. Kuo, A. Hamma, D. A. Lidar, and P. Zanardi, Quantum Adiabatic Brachistochrone, Phys. Rev. Lett. 103, 080502 (2009).

[23] A. T. Rezakhani, D. F. Abasto, D. A. Lidar, and P. Zanardi, Intrinsic geometry of quantum adiabatic evolution and quantum phase transitions, Phys. Rev. A 82, 012321 (2010). 
[24] K. Takahashi, Hamiltonian engineering for adiabatic quantum computation: Lessons from shortcuts to adiabaticity, J. Phys. Soc. Jpn. 88, 061002 (2019).

[25] S. Jansen, M.-B. Ruskai, and R. Seiler, Bounds for the adiabatic approximation with applications to quantum computation, J. Math. Phys. 48, 102111 (2007).

[26] D. A. Lidar, A. T. Rezakhani, and A. Hamma, Adiabatic approximation with exponential accuracy for many-body systems and quantum computation, J. Math. Phys. 50, 102106 (2009).

[27] S. Boixo and R. D. Somma, Necessary condition for the quantum adiabatic approximation, Phys. Rev. A 81, 032308 (2010).

[28] A. Elgart and G. A. Hagedorn, A note on the switching adiabatic theorem, J. Math. Phys. 53, 102202 (2012).

[29] M. Demirplak and S. A. Rice, Adiabatic population transfer with control fields, J. Phys. Chem. A 107, 9937 (2003).

[30] M. Demirplak and S. A. Rice, Assisted adiabatic passage revisited, J. Phys. Chem. B 109, 6838 (2005).

[31] M. V. Berry, Transitionless quantum driving, J. Phys. A 42, 365303 (2009).

[32] X. Chen, A. Ruschhaupt, S. Schmidt, A. del Campo, D. GuéryOdelin, and J. G. Muga, Fast Optimal Frictionless Atom Cooling in Harmonic Traps: Shortcut to Adiabaticity, Phys. Rev. Lett. 104, 063002 (2010).

[33] E. Torrontegui, S. Ibáñez, S. Martínez-Garaot, M. Modugno, A. del Campo, D. Guéry-Odelin, A. Ruschhaupt, X. Chen, and J. G. Muga, Shortcuts to adiabaticity, Adv. At., Mol., Opt. Phys. 62, 117 (2013).

[34] D. Guéry-Odelin, A. Ruschhaupt, A. Kiely, E. Torrontegui, S. Martínez-Garaot, and J. G. Muga, Shortcuts to adiabaticity: Concepts, methods, and applications, Rev. Mod. Phys. 91, 045001 (2019).

[35] A. C. Santos and M. S. Sarandy, Superadiabatic controlled evolutions and universal quantum computation, Sci. Rep. 5, 15775 (2015).

[36] I. B. Coulamy, A. C. Santos, I. Hen, and M. S. Sarandy, Energetic cost of superadiabatic quantum computation, Front. ICT 3, 19 (2016).

[37] S. Campbell and S. Deffner, Trade-Off Between Speed and Cost in Shortcuts to Adiabaticity, Phys. Rev. Lett. 118, 100601 (2017).

[38] K. Funo, J.-N. Zhang, C. Chatou, K. Kim, M. Ueda, and A. del Campo, Universal Work Fluctuations During Shortcuts to Adiabaticity by Counterdiabatic Driving, Phys. Rev. Lett. 118, 100602 (2017).

[39] C. Jarzynski, Generating shortcuts to adiabaticity in quantum and classical dynamics, Phys. Rev. A 88, 040101(R) (2013).
[40] A. del Campo, Shortcuts to Adiabaticity by Counterdiabatic Driving, Phys. Rev. Lett. 111, 100502 (2013).

[41] S. Deffner, C. Jarzynski, and A. del Campo, Classical and Quantum Shortcuts to Adiabaticity for Scale-Invariant Driving, Phys. Rev. X 4, 021013 (2014).

[42] M. Okuyama and K. Takahashi, From Classical Nonlinear Integrable Systems to Quantum Shortcuts to Adiabaticity, Phys. Rev. Lett. 117, 070401 (2016).

[43] D. Sels and A. Polkovnikov, Minimizing irreversible losses in quantum systems by local counterdiabatic driving, Proc. Natl. Acad. Sci. U.S.A. 114, E3909 (2017).

[44] A. B. Özgüler, R. Joynt, and M. G. Vavilov, Steering random spin systems to speed up the quantum adiabatic algorithm, Phys. Rev. A 98, 062311 (2018).

[45] H. R. Lewis and W. B. Riesenfeld, An exact quantum theory of the time-dependent harmonic oscillator and of a charged particle in a time-dependent electromagnetic field, J. Math. Phys. (NY) 10, 1458 (1969).

[46] K. Takahashi, Shortcuts to adiabaticity applied to nonequilibrium entropy production: An information geometry viewpoint, New J. Phys. 19, 115007 (2017).

[47] K. Takahashi, Unitary deformations of counterdiabatic driving, Phys. Rev. A 91, 042115 (2015).

[48] S. Morita, Faster annealing schedules for quantum annealing, J. Phys. Soc. Jpn. 76, 104001 (2007).

[49] T. Albash and D. A. Lidar, Decoherence in adiabatic quantum computation, Phys. Rev. A 91, 062320 (2015).

[50] P. W. Anderson, Infrared Catastrophe in Fermi Gases with Local Scattering Potentials, Phys. Rev. Lett. 18, 1049 (1967).

[51] T. Fogarty, S. Deffner, T. Busch, and S. Campbell, Orthogonality Catastrophe as a Consequence of the Quantum Speed Limit, Phys. Rev. Lett. 124, 110601 (2020).

[52] M. Heyl, A. Polkovnikov, and S. Kehrein, Dynamical Quantum Phase Transitions in the Transverse-Field Ising Model, Phys. Rev. Lett. 110, 135704 (2013).

[53] Y. Aharonov, D. Z. Albert, and L. Vaidman, How the Result of a Measurement of a Component of the Spin of a Spin-1/2 Particle Can Turn Out to be 100, Phys. Rev. Lett. 60, 1351 (1988).

[54] P. Jordan and E. Wigner, Über das Paulische Äquivalenzverbot, Z. Phys. A 47, 631 (1928).

[55] E. Lieb, T. Schultz, and D. Mattis, Two soluble models of an antiferromagnetic chain, Ann. Phys. (NY) 16, 407 (1961).

[56] T. Obuchi, S. Suzuki, and K. Takahashi, Complex semiclassical analysis of the Loschmidt amplitude and dynamical quantum phase transitions, Phys. Rev. B 95, 174305 (2017). 